Research Article

\title{
Identification and Screening of Natural Neuraminidase Inhibitors from Reduning Injection via One-Step High-Performance Liquid Chromatography-Fraction Collector and UHPLC/Q-TOF-MS
}

\author{
Lijun An, ${ }^{1,2}$ Xie-An Yu, ${ }^{1,2,3}$ Wei Liu, ${ }^{1,2}$ Jin Li $\mathbb{D}^{1},{ }^{1}$ and Yan-Xu Chang $\mathbb{D}^{1,2}$ \\ ${ }^{1}$ Tianjin State Key Laboratory of Modern Chinese Medicine, Tianjin University of Traditional Chinese Medicine, \\ Tianjin 300193, China \\ ${ }^{2}$ Tianjin Key Laboratory of Phytochemistry and Pharmaceutical Analysis, Tianjin University of Traditional Chinese Medicine, \\ Tianjin 300193, China \\ ${ }^{3}$ Shenzhen Institute for Drug Control, Shenzhen 518057, China \\ Correspondence should be addressed to Jin Li; lijin@tjutcm.edu.cn and Yan-Xu Chang; tcmcyx@126.com
}

Received 3 August 2020; Revised 22 October 2020; Accepted 30 October 2020; Published 17 November 2020

Academic Editor: Neil D. Danielson

Copyright (c) 2020 Lijun An et al. This is an open access article distributed under the Creative Commons Attribution License, which permits unrestricted use, distribution, and reproduction in any medium, provided the original work is properly cited.

\begin{abstract}
Neuraminidase plays an essential role in the spread of influenza viruses via cleaving sialic acids from the host cell receptors and virions. Neuraminidase has been regarded as an essential target for prevention and treatment of influenza infection. The one-step high-performance liquid chromatography-fraction collector (HPLC-FC) was selected to prepare fractions from Reduning (RDN) injection, while ultra-high-performance liquid chromatography/quadrupole-time-of-flight mass spectrometry (UHPLC/Q-TOFMS) was used to identify fractions depending on their retention time and molecular ion. As a result, 75 fractions were prepared and 28 fractions out of them exhibited NA inhibitory effects with the dose-effect relationship. Exploring it further, six components including neochlorogenic acid, chlorogenic acid, cryptochlorogenic acid, isochlorogenic acid B, isochlorogenic acid A, and isochlorogenic acid $\mathrm{C}$ were the main components that accounted for almost $80 \%$ of inhibitory activity of RDN injection. Accordingly, these results demonstrated that this strategy could not only rapidly identify but also accurately screen active components from traditional Chinese medicine.
\end{abstract}

\section{Introduction}

Influenza virus is still a threat to human lives. It possesses two major glycoproteins enzymes, hemagglutinin (HA) and neuraminidase (NA) $[1,2]$. HA binds to the host cell receptors to facilitate viral entry, while NA facilitates the spread of the infection by releasing newly synthesized virions into neighboring cells [3]. Replication, infection, and spreading play a key role in the pathogenesis of the influenza virus, especially for spreading. Accumulative evidences demonstrated that NA played an essential role in the spread of influenza viruses by cleaving sialic acids from the host cell receptors and virions [4, 5]. Therefore, NA has been regarded as an essential target for prevention and treatment of influenza infection. Besides, NA could also be used as an important candidate antigen for universal influenza vaccines [6]. Zanamivir and oseltamivir, as NA inhibitors (NAIs), are the most common drugs for combating the influenza virus. However, drug-resistant virus of these drugs has emerged [7]. Moreover, oseltamivir is expensive and zanamivir cannot to be taken orally. Accordingly, with an increasing appearance on the drug resistance of antiviral drug [8-10], screening novel NAIs from Traditional Chinese Medicine (TCM) has attracted great attention towards the treatment of influenza.

Traditional Chinese Medicines (TCMs) played important roles in clinical practice over the past years. It has been widely accepted by most countries owing to its long-term 
clinical use and reliable efficacy. However, there are still some challenges for exploring the pharmacology function of TCM because the chemical structures of active ingredients are largely undefined. In general, traditional methods for screening active components have relied on animal and cell models, which was complex to operation and time-consuming. Hence, it was essential to establish a rapid, convenient, and accurate method to screen and identify the bioactive components in TCM.

Reduning injection (RDN) contains some effective ingredients extracted from three herbs (Flos Lonicerae, Herba Artemisiae annuae, and Gardenia jasminoides). It has been widely used as an antipyretic and anti-inflammatory drug in China [11]. In clinic, RDN injection not only has been used to treat common cold, cough, acute upper respiratory infection, and acute bronchitis, but also has a potential therapeutic effect on influenza. A novel online DPPH-CEDAD method [12] was developed for quality assessment of RDN injection and a reliable pharmacology model [13] was constructed for understanding the molecular mechanisms of multiple components from RND injection, respectively. The pharmacological target at signaling pathways level and the potential target proteins and the key pathways of RDN injection have also been investigated [14, 15]. However, there is no report on screening and identification of NAIs from RDN injection. Furthermore, a great number of researchers paid attention to screening active components from TCM. Ge et al. [16] established an activity-integrated strategy to screen glucosidase inhibitors from Coptis chinensis and $\mathrm{Lu}$ et al. [17] developed a peak fractionation approach to screen direct thrombin inhibitors from Radix Salvia miltiorrhiza, respectively [18]. Taking these methods into consideration, there was little literature to screen NA inhibitors from RDN injection and summarize the main active components for elucidating the effective substances of $\mathrm{RDN}$ injection.

In this study, an accurate strategy for preparing, screening, and identification of NAIs from RDN injection was developed based on NA inhibitory activity. The 75 fractions were prepared by high-performance liquid chromatography-fraction collector (HPLC-FC) in one run depending on the retention time. Following that, ultrahigh-performance liquid chromatography (UHPLC) and ultra-high-performance liquid chromatography/quadrupole-time-of-flight mass spectrometry (UHPLC/Q-TOFMS) were used for the precise identification of each fraction and NA inhibitory activities of all fractions were performed with the same time. In total, 67 components were identified from RDN injection and 15 active components with an inhibitory effect against NA were screened and identified. It was found that neochlorogenic acid, chlorogenic acid, cryptochlorogenic acid, isochlorogenic acid B, isochlorogenic acid $\mathrm{A}$, and isochlorogenic acid $\mathrm{C}$ were the main active components that accounted for $80 \%$ of inhibitory activity among the RDN injection. Accordingly, the one-step work can not only identify 67 components from RDN injection, but also screen natural NAIs from TCM and provides a promising strategy for elucidating the effective substances of TCM.

\section{Materials and Methods}

2.1. Chemicals and Materials. Reduning injections were offered from Jiangsu Kanion Pharmaceutical Co., Ltd. (Lianyungang, China). Acetonitrile (Dikma Technologies Inc., USA), methanol (Tianjin Concord Science Co., Ltd, Tianjin, China), and formic acid (Tianjin Kermel Science Company) were of HPLC grade. Deionized water was purified with a Milli-Q Academic ultrapure water system (Millipore, Milford, MA, USA) and reference standards including neochlorogenic acid, chlorogenic acid, cryptochlorogenic acid, isochlorogenic acid $\mathrm{B}$, isochlorogenic acid $\mathrm{A}$, and isochlorogenic acid $\mathrm{C}$ were purchased from Chengdu Must Biotechnology Co., Ltd. (Chengdu, China). Neuraminidase inhibitors screen kits were also purchased from Beyotime Biotechnology.

\subsection{Chromatographic Conditions. An Agilent 1260 HPLC} system (Agilent Technologies, Germany) equipped with a binary pump, an online degasser, an autosampler, a column oven, and a variable wavelength detector (DAD) was used to separate the multiple components from RDN injection. The separation was achieved on Agilent $\mathrm{C}_{18}$ column $(4.6 \times 250 \mathrm{~mm}, 5 \mu \mathrm{m})$. The mobile phase consisted of $0.1 \%(\mathrm{v} /$ v) formic acid aqueous solution (A) and acetonitrile (B) using a gradient elution of $0-15 \mathrm{~min}, 5-5 \% \mathrm{~B} ; 15-20 \mathrm{~min}$, 5-6\% B; 20-50 min, 6-6\% B; 50-55 min, 6\%-15\% B; 55-60 min, $15 \%-18 \%$ B; 60-65 min, $18 \%-25 \%$ B; $65-70$ min, $25 \%-35 \% \mathrm{~B}$; and $70-75 \mathrm{~min}, 35 \%-90 \% \mathrm{~B}$. The flow rate was

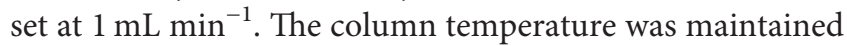
at $30^{\circ} \mathrm{C}$. The injection volume was $10 \mu \mathrm{L}$ and detection wavelength was set at 225 and $325 \mathrm{~nm}$.

A Waters ACQUITY UHPLC System (Waters Co., Milford, MA) equipped with a photodiode array detector was used in the range of $190-400 \mathrm{~nm}$. The system was controlled by Empower 2 workstation. An ACQUITY UHPLC BEH C ${ }_{18}(1.7 \mu \mathrm{m}, 2.1 \times 50 \mathrm{~mm})$ column was used for separation with the mobile phase $0.1 \%(\mathrm{v} / \mathrm{v})$ formic acid aqueous solution (A) and acetonitrile (B) using a gradient elution: $0-4 \mathrm{~min}, 4-4 \% \mathrm{~B}$; 4-6 $\mathrm{min}, 4-5 \% \mathrm{~B}$; 6-9 $\mathrm{min}, 5-7 \%$ B; 9-11 min, 7-9\% B; 11-12 min, 9-12\% B; $12-13 \mathrm{~min}$, $12-14 \% \mathrm{~B} ; 13-15 \mathrm{~min}, 14-15 \% \mathrm{~B} ; 15-16 \mathrm{~min}, 15-16 \% \mathrm{~B}$; $16-18 \mathrm{~min}, 16-19 \% \mathrm{~B} ; 18-19 \mathrm{~min}, 19-20 \% \mathrm{~B} ; 19-20 \mathrm{~min}$, $20-30 \% \mathrm{~B} ; 20-22 \mathrm{~min}, 30-50 \% \mathrm{~B} ; 22-23 \mathrm{~min}, 50-95 \% \mathrm{~B}$; and 23-24 min, 95-4\% B. The flow rate of mobile phase was set at $0.3 \mathrm{~mL} \mathrm{m^{-1 }}$ and the column temperature and sample temperature were set at $40^{\circ} \mathrm{C}$ and $10^{\circ} \mathrm{C}$, respectively. The injection volume was $1 \mu \mathrm{L}$. The detection wavelengths were set at $225 \mathrm{~nm}$ and $325 \mathrm{~nm}$, respectively.

The identification of each fraction of RDN injection was performed by an Agilent UHPLC-Q-TOF/MS system. The ESI-MS spectra were acquired in the negative ion mode. The capillary voltage was set to $3.0 \mathrm{kV}$ for the negative ion mode, high-purity nitrogen was set as the nebulization, and auxiliary gas with the gas pressure was 35 psig. The velocity of

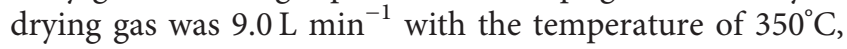
the fragmentor voltage was at $175 \mathrm{~V}$, skimmer voltage was $65 \mathrm{~V}$, and the collision energy (CE) was at 30, respectively. 
Data was collected at the scan range of 100-1500 Da for MS and 50-1500 Da for MS/MS. Data acquisition, exact mass, and elemental composition analyses were controlled by Mass Hunter software (Agilent Technologies). The mobile phase, column, column temperature, flow rate, and gradient program used in the UHPLC-Q-TOF/MS analysis were the same as the UHPLC analysis.

2.3. Preparation of Sample Solutions. An Agilent series 1260 HPLC system with Fraction collector (BSZ-100, Shanghai Qingpu Huxi Instrument, Shanghai, China) was used for the fractions collection. The RDN injection was injected into the HPLC system for the separation of each compound. Fractions were automatically collected by using a fixed time of $1 \mathrm{~min}$. As a result, a total of 75 fractions were prepared. They were evaporated to dryness by the nitrogen gas and the residues were redissolved and diluted for the activity assay. Thus, the sample solutions for the bioassays were obtained. In order to screen the NA inhibition effects with dose-effect relationship, three different concentrations (low, medium, and high concentration) were prepared for activity assay. Therefore, the dried residue was reconstituted in $150 \mu \mathrm{L}$ water, $75 \mu \mathrm{L}$ was for activity assay (high concentration), $75 \mu \mathrm{L}$ was diluted with $75 \mu \mathrm{L}$ water (medium concentration), and finally the low concentration was diluted with $75 \mu \mathrm{L}$ water.

2.4. Bioassay of NA Inhibitors. $70 \mu \mathrm{L}$ buffer solution was added to a 96-well plate and different doses $(0,1,2,5,7.5$, and $10 \mu \mathrm{L}$ ) of NA were mixed with it. Then different volumes $(20,19,18,15,12.5$, and 10) $\mu \mathrm{L}$ of Milli-Q water were added to reach the total volume to $90 \mu \mathrm{L}$, respectively. It was then incubated for $2 \mathrm{~min}$ at $37^{\circ} \mathrm{C}$ and then $10 \mu \mathrm{L}$ of NA fluorescence substrate was added into the plate, incubating for $30 \mathrm{~min}$ before detection. The fluorescence value (FV) of wells was measured at $360 \mathrm{~nm}$ of excitation wavelength and $450 \mathrm{~nm}$ of emission wavelength by Flex Station 3 Microplate Reader. The relationship between the dose of NA and FV is stated below:

$$
\mathrm{FV}=a \log (\text { dose of } \mathrm{NA})+b .
$$

$70 \mu \mathrm{L}$ buffer of solution was added to a 96-well plate. $10 \mu \mathrm{L}$ of NA and $10 \mu \mathrm{L}$ of each fraction were added into it. The volume was then adjusted to $90 \mu \mathrm{L}$ with $10 \mu \mathrm{L}$ of Milli-Q water. After incubated for $2 \mathrm{~min}$ at $37^{\circ} \mathrm{C}, 10 \mu \mathrm{L}$ of $\mathrm{NA}$ fluorescence substrate was then added into the plate, incubating for $30 \mathrm{~min}$ before detection. The inhibition rate of each fraction was calculated by the following formula:

inhibition percentage $=\frac{(10-10)^{([(\mathrm{FV} f m-\mathrm{FV} f b)-b] / a)}}{10} * 100 \%$,

where FV fm represented the FV of each fraction and FV fb represented the blank background of each fraction.

\section{Result and Discussion}

3.1. Optimization of Chromatographic Conditions. The HPLC conditions were considered for obtaining chromatograms with better resolution. The different mobile phases (acetonitrile-water, methanol-water), concentrations of additive $(0.1 \%, 0.2 \%$, and $0.3 \%$ formic acid and phosphoric acid), and column temperatures $\left(20,30\right.$, and $\left.40^{\circ} \mathrm{C}\right)$ were optimized to achieve better separation. By comparing resolutions and the peak shapes of the investigated components, the best separation was achieved when acetonitrilewater was selected as mobile phase and $0.1 \%$ formic acid was chosen as an additive to the water phase. The column temperature and flow rate were optimal at $40^{\circ} \mathrm{C}$ and $1 \mathrm{~mL}$ $\mathrm{min}^{-1}$, respectively. The HPLC chromatogram is shown in Figure 1.

The UHPLC conditions were also considered as a requirement for obtaining chromatograms with better peak resolution in the same way as the HPLC. But the flow rate was set as $0.3 \mathrm{~mL} \mathrm{~min}{ }^{-1}$. The UHPLC chromatogram is displayed in Figure 2.

\subsection{Identification of 67 Components in RDN Injection Using} UHPLC/Q-TOF-MS. The peak number, retention time, MS/MS fragmentation ions information, and identification of 67 components are listed in Table 1 . Peak 1 was of molecular weight 192.0634 with ions of $\mathrm{m} / \mathrm{z} 191.0558$ $\left(\mathrm{C}_{7} \mathrm{H}_{11} \mathrm{O}_{6}\right)[\mathrm{M}-\mathrm{H}]^{-}$and $111.0445\left(\mathrm{C}_{6} \mathrm{H}_{7} \mathrm{O}_{2}\right)\left[\mathrm{M}-\mathrm{H}-\mathrm{CO}_{2^{-}}\right.$ $\left.2 \mathrm{H}_{2} \mathrm{O}\right]^{-}$was observed in MS/MS spectra. Therefore, it was identified as quinic acid [19]. Peak 4 had the major firstorder mass spectrum at $\mathrm{m} / \mathrm{z} 353.0875\left(\mathrm{C}_{16} \mathrm{H}_{18} \mathrm{O}_{9}\right)[\mathrm{M}-\mathrm{H}]^{-}$ and the MS/MS fragments were at $191.0554\left(\mathrm{C}_{7} \mathrm{H}_{11} \mathrm{O}_{6}\right)[\mathrm{M}-$ $\left.\mathrm{H}-\mathrm{C}_{6} \mathrm{H}_{12} \mathrm{O}_{6}+\mathrm{H}_{2} \mathrm{O}\right]^{-}, \quad 179.0344 \quad\left(\mathrm{C}_{9} \mathrm{H}_{7} \mathrm{O}_{4}\right), \quad 161.0237$ $\left(\mathrm{C}_{9} \mathrm{H}_{5} \mathrm{O}_{3}\right) \quad\left[\mathrm{C}_{9} \mathrm{H}_{7} \mathrm{O}_{4}-\mathrm{H}_{2} \mathrm{O}\right]^{-}$, and $135.0447 \quad\left(\mathrm{C}_{8} \mathrm{H}_{7} \mathrm{O}_{2}\right)$ $\left[\mathrm{C}_{9} \mathrm{H}_{7} \mathrm{O}_{4}-\mathrm{CO}_{2}\right]^{-}$, which could be speculated to be an organic acid. Peak 19 had a molecular weight of $179.0351\left(\mathrm{C}_{9} \mathrm{H}_{7} \mathrm{O}_{4}\right)$ with a fragment of $135.045\left(\mathrm{C}_{8} \mathrm{H}_{7} \mathrm{O}_{2}\right)\left[\mathrm{C}_{9} \mathrm{H}_{7} \mathrm{O}_{4}-\mathrm{CO}_{2}\right]^{-}$and therefore was identified as caffeic acid [20]. Based on the same regular, peaks $4,5,10,16,20,22,25,33,41,58,61,62$, 63 , and 65 were identified as two types of isomers 353.0875 $\left(\mathrm{C}_{16} \mathrm{H}_{18} \mathrm{O}_{9}\right)$ and $515.1197\left(\mathrm{C}_{25} \mathrm{H}_{24} \mathrm{O}_{12}\right)$. They were identified as cis-5-O-caffeoylquinic acid, trans-5-O-caffeoylquinic acid, trans-1-O-caffeoylquinic acid, cis-1-O-caffeoylquinic acid, cis-4-O-caffeoylquinic acid, trans-3-O-caffeoylquinic acid, trans-4-O-caffeoylquinic acid, cis-3-O-caffeoylquinic acid and 1,5-dicaffeoylquinic acid, 1,3-dicaffeoylquinic acid, 3,4-dicaffeoylquinicacid, 3,5-dicaffeoylquinicacid, 4,5dicaffeoylquinicacid, and 1,4-dicaffeoylquinicacid depending on their different retention times [20-26]. The mass spectrum of peak 7 with retention time of 2.93 min offered an $[\mathrm{M}-\mathrm{H}]^{-}$ion at $\mathrm{m} / \mathrm{z}$ 451.0599. Characteristic product ions $243.0861\left[\mathrm{M}-\mathrm{H}-\mathrm{C}_{6} \mathrm{H}_{12} \mathrm{O}_{6}-\mathrm{CO}\right]^{-}, 191.0547\left(\mathrm{C}_{7} \mathrm{H}_{11} \mathrm{O}_{6}\right)$, and $179.0349\left(\mathrm{C}_{9} \mathrm{H}_{7} \mathrm{O}_{4}\right)$ were the special ions of an organic acid. Thus, this compound could be speculated as ixoroside [27]. Peaks 24, 38, and 41 exhibited the same $[\mathrm{M}-\mathrm{H}]^{-}$ion at $\mathrm{m} / \mathrm{z}$ 367.1038, while 191.0546 and 173.0344 were organic acid special fragment. The MS/MS spectrum produced 191.0546 $\left[\mathrm{M}-\mathrm{H}-\mathrm{CH}_{2}-\mathrm{C}_{6} \mathrm{H}_{12} \mathrm{O}_{6}+\mathrm{H}_{2} \mathrm{O}\right]^{-}$and 173.0344 [M-H- $\mathrm{CH}_{2}-$ 


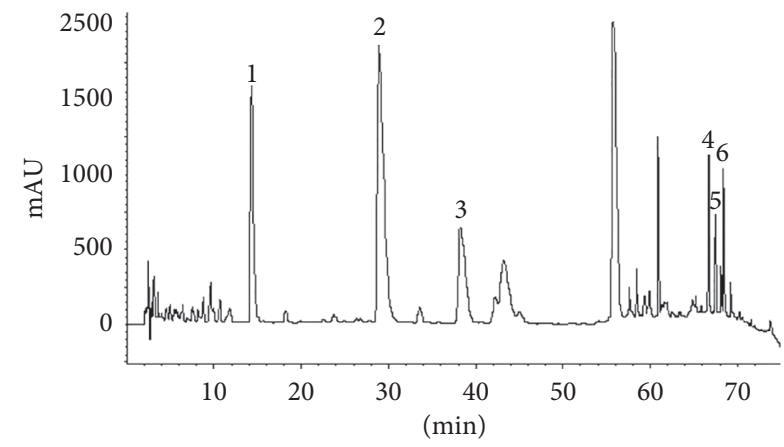

FIgURE 1: HPLC chromatogram of RDN injection. 1: neochlorogenic acid; 2: chlorogenic acid; 3: cryptochlorogenic acid; 4: isochlorogenic acid $\mathrm{B}$; 5: isochlorogenic acid $\mathrm{A}$; and 6: isochlorogenic acid C.



FIgURE 2: UHPLC chromatogram of RDN injection. 1: neochlorogenic acid; 2: chlorogenic acid; 3: cryptochlorogenic acid; 4: isochlorogenic acid $\mathrm{B}$; 5: isochlorogenic acid $\mathrm{A}$; and 6: isochlorogenic acid C.

$\left.\mathrm{C}_{6} \mathrm{H}_{12} \mathrm{O}_{6}\right]^{-}$, from which it can be speculated that $\mathrm{CH}_{2}$ has been added to caffeoylquinic acid. Therefore, peaks 24,38 , and 41 were identified as 5-O-caffeoylqunic methyl ester, 4O-caffeoylqunic methyl ester, and 3-O-caffeoylqunic methyl ester [28]. Peak 47 had the special fragment ion at 367.1019 and product ion of 173.045 was obtained by losing $\mathrm{C}_{6} \mathrm{H}_{12} \mathrm{O}_{6}$ in MS/MS spectrum. Therefore, it was identified as 3,5 -di-O-caffeoylquinic methyl ester [20,27]. Peak 65 in the MS/MS experiment gave fragmentation information at $353.0869,191.0543$, and 161.0439, which can be regarded as organic acid. The first-order mass spectrum ion of 659.1607 $[\mathrm{M}-\mathrm{H}]$ was treated as the base peak. The ion at $497.1296 \mathrm{can}$ be formed through $\left[\mathrm{M}-\mathrm{H}-\mathrm{C}_{6} \mathrm{H}_{12} \mathrm{O}_{6}+\mathrm{H}_{2} \mathrm{O}\right]$ and 353.0877 through $\left[\mathrm{M}-\mathrm{H}-2 \mathrm{C}_{6} \mathrm{H}_{12} \mathrm{O}_{6}+3 \mathrm{H}_{2} \mathrm{O}\right]$. Hence, 3-O-(3-hydroxy, 3-methyl)glutaroyl-4-O-caffeoylquinic acid [22], 3O-caffeoyl-4-O-(3-hydroxy, 3-methyl)glutaroylquinic acid [29], and 3,5-di-O-Caffeoyl-4-O-(3-hydroxy-3-methyl)glutaroylquinic acid [22] were therefore identified. The same pattern was with peak 60 weighed 723.2134 $\left(\mathrm{C}_{33} \mathrm{H}_{40} \mathrm{O}_{18}\right)$ and therefore it was suspected to be Jasmigeniposide A [22]. Peaks 14 and 35 had the first-order mass spectrum ion of $337.0932[\mathrm{M}-\mathrm{H}]$, the special fragment ions of $191.0555\left[\mathrm{M}-\mathrm{H}-\mathrm{C}_{9} \mathrm{H}_{6} \mathrm{O}_{2}\right]^{-}, 119.0503\left[\mathrm{M}-\mathrm{H}-\mathrm{C}_{9} \mathrm{H}_{6} \mathrm{O}_{2^{-}}\right.$
$\left.\mathrm{H}_{2} \mathrm{O}-\mathrm{CO}_{2}\right]^{-}$, and 163.039 [M- $\left.\mathrm{H}-\mathrm{C}_{9} \mathrm{H}_{6} \mathrm{O}_{2}-\mathrm{H}_{2} \mathrm{O}\right]^{-}$. Based on their different retention times of 4.68 and 10.8 , they were identified as 3-p-coumaroylquinic acids and 5-p-coumaroylquinic acids [30]. Peaks 2, 17, and 29 offered the same $[\mathrm{M}-\mathrm{H}]^{-}$ion at the $\mathrm{m} / \mathrm{z} 373.1137\left(\mathrm{C}_{16} \mathrm{H}_{22} \mathrm{O}_{10}\right)$. In the MS/MS spectrum, they all had the characteristic product ions at $\mathrm{m} / \mathrm{z}$ $211.06 \quad\left(\mathrm{C}_{10} \mathrm{H}_{11} \mathrm{O}_{5}\right) \quad\left[\mathrm{M}-\mathrm{C}_{6} \mathrm{H}_{12} \mathrm{O}_{6}+\mathrm{H}_{2} \mathrm{O}\right]^{-}, \quad 193.0496$

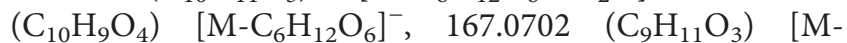
$\left.\mathrm{C}_{6} \mathrm{H}_{12} \mathrm{O}_{6}-\mathrm{CO}_{2}+\mathrm{H}_{2} \mathrm{O}\right]^{-}, 149.0602\left(\mathrm{C}_{9} \mathrm{H}_{9} \mathrm{O}_{2}\right)\left[\mathrm{M}-\mathrm{C}_{6} \mathrm{H}_{12} \mathrm{O}_{6^{-}}\right.$ $\left.\mathrm{CO}_{2}\right]^{-}$, and $123.0445 \quad\left(\mathrm{C}_{7} \mathrm{H}_{7} \mathrm{O}_{2}\right) \quad\left[\mathrm{M}-\mathrm{C}_{6} \mathrm{H}_{12} \mathrm{O}_{6}-\right.$ $\left.2 \mathrm{CO}_{2}+\mathrm{H}_{2} \mathrm{O}\right]^{-}$, resulting from losses of glucose, $\mathrm{H}_{2} \mathrm{O}$, and $\mathrm{CO}_{2}$. Depending on the difference in their retention times at $1.53,4.97$, and 9.1, they were tentatively identified as geniposidic acid [31], secologanic acid [32], and gardoside [21]. In the first-order mass spectrum of peak 3 , the $[\mathrm{M}-\mathrm{H}]^{-}$ ion at the $\mathrm{m} / \mathrm{z} 391.1241\left(\mathrm{C}_{16} \mathrm{H}_{24} \mathrm{O}_{11}\right)$ produced the MS/MS ions as 229.0705, 211.0596, 185.081, 167.0694, and 149.0596 due to the loss of $\mathrm{C}_{6} \mathrm{H}_{12} \mathrm{O}_{6}, \mathrm{CO}_{2}$, and $\mathrm{H}_{2} \mathrm{O}$, which can be identified as shanzhiside [21]. By the similar regular pattern of confirming the fragmentation, peaks $9,12,13,18,23,28$, $31,32,35,38,47,53,56,59,60,68,70$, and 71 were identified as mussaenosidic acid, shanzhiside methyl ester, scandoside methyl ester, gardenoside, isoshanzhiside methyl ester, deacetylasperulosidic acid methyl ester, genipin gentiobioside, genipin-1b-gentiobioside, geniposide, gardenoside, $6^{\prime}$-O-trans-coumaroyl geniposidic acid, quercetin-3-Oglucoside, luteolin-7-O-D-glucopyranoside, 6-O-transcoumaroyl geniposidic, 10-acetyl geniposide, $6^{\prime}$-O-transcoumaroyl genipin gentiobioside, 6"-O-trans-sinapoyl genipin gentiobioside, and 6"-O-trans-cinnamoyl genipin gentiobioside $[19,21,22,27,33-36]$. Peak 8 showed the mass spectrum ion at $\mathrm{m} / \mathrm{z} 345.1291\left(\mathrm{C}_{16} \mathrm{H}_{25} \mathrm{O}_{8}\right)[\mathrm{M}-\mathrm{H}]^{-}$. The product ions $165.0911\left[\mathrm{M}-\mathrm{H}-\mathrm{C}_{6} \mathrm{H}_{12} \mathrm{O}_{6}\right]^{-}$and 121.1022 [M- $\left.\mathrm{H}-\mathrm{C}_{6} \mathrm{H}_{12} \mathrm{O}_{6}-\mathrm{CO}_{2}\right]^{-}$were supported to surmise it as jasminoside [27]. Peak 11 had the major ions at $\mathrm{m} / \mathrm{z}$ $183.1025[\mathrm{M}-\mathrm{H}]^{-}$and the MS/MS fragments were 139.1125 $\left[\mathrm{M}-\mathrm{H}-\mathrm{CO}_{2}\right]^{-}$and $121.0956\left[\mathrm{M}-\mathrm{H}-\mathrm{CO}_{2}-\mathrm{H}_{2} \mathrm{O}\right]^{-}$. Therefore, it was identified as jasminodiol [27]. Peaks 21 and 26 were a type of isomer which yielded the same ion at 375.1291 [M$\mathrm{H}]^{-}$in the first-order mass spectrum. They had the same prominent ion at $213.0765\left[\mathrm{M}-\mathrm{H}-\mathrm{C}_{6} \mathrm{H}_{12} \mathrm{O}_{6}+\mathrm{H}_{2} \mathrm{O}\right]^{-}$and $151.0761\left[\mathrm{M}-\mathrm{H}-\mathrm{C}_{6} \mathrm{H}_{12} \mathrm{O}_{6}-\mathrm{CO}_{2}\right]^{-}$. But they were speculated to be loganic acid [37] and 8-epi-loganic acid based on their retention times. Peak 40 gave a mass spectrum ion at $375.1667\left(\mathrm{C}_{17} \mathrm{H}_{27} \mathrm{O}_{9}\right)$ with MS/MS fragments of 285.1087, 193.0491, 173.0457, and 135.0435, which were detected to be secologanic acid [20]. Peak 28 showed the mass spectrum ion at $389.108[\mathrm{M}-\mathrm{H}]^{-}$. The product fragment ions at $345.1166 \quad\left[\mathrm{M}-\mathrm{H}-\mathrm{CO}_{2}\right]^{-}, \quad 209.0438 \quad\left[\mathrm{M}-\mathrm{H}-\mathrm{C}_{6} \mathrm{H}_{12} \mathrm{O}_{6}\right]^{-}$, $183.0653\left[\mathrm{M}-\mathrm{H}-\mathrm{C}_{6} \mathrm{H}_{12} \mathrm{O}_{6}-\mathrm{H}_{2} \mathrm{O}\right]^{-}$, and 139.0066 [M-H$\left.\mathrm{C}_{6} \mathrm{H}_{12} \mathrm{O}_{6}-\mathrm{H}_{2} \mathrm{O}-\mathrm{CO}_{2}\right]^{-}$constituted the structure of deacetyl asperulosidic acid [27]. Peaks 48 and 51 gave the $[\mathrm{M}-\mathrm{H}]^{-}$ion at the $\mathrm{m} / \mathrm{z} 279.123$ and 281.1389. The fragments of peak 51 were 205.1219 [M-H-HCOOH-CO] $]^{-}$and 187.1107 [M-H$\left.\mathrm{HCOOH}-\mathrm{CO}-\mathrm{H}_{2} \mathrm{O}\right]^{-}$. Thus, artemisitene was detected at peak of 51, and peak 48 was identified as artemisitene [38]. Peak 33 was detected with precursor ions at $\mathrm{m} / \mathrm{z} 281.1386$ $[\mathrm{M}-\mathrm{H}]^{-}$, which produced MS/MS base peak ion at $\mathrm{m} / \mathrm{z}$ $237.1456\left[\mathrm{M}-\mathrm{H}-\mathrm{C}_{6} \mathrm{H}_{12} \mathrm{O}_{6}-\mathrm{CO}_{2}\right]^{-}$and $201.127 \quad[\mathrm{M}-\mathrm{H}-$ 
TABLE 1: UPLC/Q-TOF-MS data and identification 67 compounds in RDN injection.

\begin{tabular}{|c|c|c|c|c|c|c|}
\hline $\begin{array}{l}\text { Peak } \\
\text { no. }\end{array}$ & $\begin{array}{l}\mathrm{RT} \\
(\mathrm{min})\end{array}$ & {$[\mathrm{M}-\mathrm{H}]^{-}$} & MS/MS fragmentation ions & Formula & Identification & ppm \\
\hline 1 & 0.71 & 191.0558 & 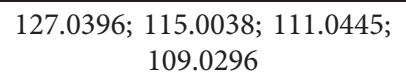 & $\mathrm{C}_{7} \mathrm{H}_{12} \mathrm{O}_{6}$ & Quinic acid & 1.62 \\
\hline 2 & 1.53 & 373.1137 & $\begin{array}{c}193.0496 ; \begin{array}{l}167.0702 ; 149.0602 \\
123.0445\end{array}\end{array}$ & $\mathrm{C}_{16} \mathrm{H}_{22} \mathrm{O}_{10}$ & Geniposidic acid & 0.86 \\
\hline 3 & 1.99 & 391.1241 & $\begin{array}{c}229.0705 ; 211.0596 ; 185.0810 \\
167.0694 ; 149.0596\end{array}$ & $\mathrm{C}_{16} \mathrm{H}_{24} \mathrm{O}_{11}$ & Shanzhiside & 1.24 \\
\hline 4 & 2.69 & 353.0875 & $191.0554 ; 179.0344 ; 135.0447$ & $\mathrm{C}_{16} \mathrm{H}_{18} \mathrm{O}_{9}$ & cis-5-O-Caffeoylquinic acid & 0.86 \\
\hline 5 & 3.05 & 353.0875 & $191.0552 ; 179.0340 ; 135.0442$ & $\mathrm{C}_{16} \mathrm{H}_{18} \mathrm{O}_{9}$ & Neochlorogenic acid & 0.86 \\
\hline 6 & 2.88 & 191.0356 & $111.0072 ; 109.0302 ; 101.9309$ & $\mathrm{C}_{10} \mathrm{H}_{8} \mathrm{O}_{4}$ & Scopoletin & -3.22 \\
\hline 7 & 2.93 & 451.0599 & $243.0861 ; 191.0547 ; 179.0349$ & $\mathrm{C}_{30} \mathrm{H}_{12} \mathrm{O}_{5}$ & Ixoroside & 2.87 \\
\hline 8 & 3.27 & 345.1552 & $165.0911 ; 121.1022 ; 101.0245$ & $\mathrm{C}_{16} \mathrm{H}_{26} \mathrm{O}_{8}$ & Jasminoside & 0.84 \\
\hline 9 & 3.52 & 375.1291 & $\begin{array}{c}213.0765 ; 169.0866 ; 151.0760 \\
125.0605\end{array}$ & $\mathrm{C}_{16} \mathrm{H}_{24} \mathrm{O}_{10}$ & Mussaenosidic acid & 1.52 \\
\hline 10 & 4.2 & 353.0875 & $191.0559 ; 179.0331 ; 135.0469$ & $\mathrm{C}_{16} \mathrm{H}_{18} \mathrm{O}_{9}$ & trans-1-O-Caffeoylquinic acid & 0.86 \\
\hline 11 & 4.43 & 183.1025 & $139.1125 ; 121.0956$ & $\mathrm{C}_{10} \mathrm{H}_{16} \mathrm{O}_{3}$ & Jasminodiol & 1.46 \\
\hline 12 & 4.58 & 405.1399 & $225.0777 ; 149.0599 ; 121.0667$ & $\mathrm{C}_{17} \mathrm{H}_{26} \mathrm{O}_{11}$ & Shanzhiside methyl ester & 0.83 \\
\hline 13 & 4.68 & 449.1295 & $\begin{aligned} 403.1258 ; & 241.0715 ; 209.0473 ; \\
& 143.0325\end{aligned}$ & $\mathrm{C}_{18} \mathrm{H}_{26} \mathrm{O}_{13}$ & Scandoside methyl ester & 1.25 \\
\hline 14 & 4.68 & 337.0932 & $191.0555 ; 163.0390 ; 119.0503$ & $\mathrm{C}_{16} \mathrm{H}_{18} \mathrm{O}_{8}$ & 3-p-Coumaroylquinic acids & -0.91 \\
\hline 15 & 4.98 & 151.0400 & 151.0400 & $\mathrm{C}_{8} \mathrm{H}_{8} \mathrm{O}_{3}$ & Vanillin & 0.45 \\
\hline 16 & 4.96 & 353.0875 & $\begin{array}{c}191.0555 ; 179.0346 ; 173.0456 ; \\
135.0451\end{array}$ & $\mathrm{C}_{16} \mathrm{H}_{18} \mathrm{O}_{9}$ & Cir-1-O-caffeoylquinic acid & 0.86 \\
\hline 17 & 4.97 & 373.1137 & $\begin{array}{c}211.0600 ; 193.0507 ; 167.0708 \\
149.0599 ; 123.0447\end{array}$ & $\mathrm{C}_{16} \mathrm{H}_{22} \mathrm{O}_{10}$ & Secologanic acid & 0.86 \\
\hline 18 & 5.3 & 449.1295 & $\begin{array}{c}241.0743 ; 139.0333 ; 121.0273 ; \\
101.0254\end{array}$ & $\mathrm{C}_{18} \mathrm{H}_{26} \mathrm{O}_{13}$ & Gardenoside & 1.52 \\
\hline 19 & 5.8 & 179.0351 & $135.0450 ; 134.0369$ & $\mathrm{C}_{9} \mathrm{H}_{8} \mathrm{O}_{4}$ & Caffeic acid & -0.65 \\
\hline 20 & 6.1 & 353.0875 & $192.0592 ; 191.1740$ & $\mathrm{C}_{16} \mathrm{H}_{18} \mathrm{O}_{9}$ & Chlorogenic acid & 0.86 \\
\hline 21 & 6.54 & 375.1291 & $\begin{aligned} 213.0765 ; & 169.0866 ; 151.0760 \\
& 125.0605\end{aligned}$ & $\mathrm{C}_{16} \mathrm{H}_{24} \mathrm{O}_{10}$ & Loganic acid & 1.52 \\
\hline 22 & 6.8 & 353.0875 & $192.0580 ; 191.0549$ & $\mathrm{C}_{16} \mathrm{H}_{18} \mathrm{O}_{9}$ & trans-3-O-Caffeoylquinic acid & 0.86 \\
\hline 23 & 6.89 & 451.1443 & $\begin{array}{c}405.1365 ; 243.0855 ; 191.0543 ; \\
119.0376\end{array}$ & $\mathrm{C}_{17} \mathrm{H}_{26} \mathrm{O}_{11}$ & Isoshanzhiside methyl ester & 3.13 \\
\hline 24 & 7.0 & 367.1038 & $191.0546 ; 149.0600 ; 134.0368$ & $\mathrm{C}_{17} \mathrm{H}_{20} \mathrm{O}_{9}$ & 5-O-Caffeoylqunic methyl ester & -0.94 \\
\hline 25 & 7.6 & 353.0875 & $191.0552 ; 179.0342 ; 135.0445$ & $\mathrm{C}_{16} \mathrm{H}_{18} \mathrm{O}_{9}$ & Cryptochlorogenic acid & 0.86 \\
\hline 26 & 8.2 & 375.1291 & $\begin{array}{c}195.0639 ; 151.0761 ; 125.0606 \\
107.0500\end{array}$ & $\mathrm{C}_{16} \mathrm{H}_{24} \mathrm{O}_{10}$ & 8-Epi-loganic acid & 1.52 \\
\hline 27 & 8.57 & 449.1294 & $\begin{array}{c}241.0676 ; 179.0539 ; 139.3100 \\
101.0242\end{array}$ & $\mathrm{C}_{18} \mathrm{H}_{26} \mathrm{O}_{13}$ & Deacetylasperulosidic acid methyl ester & 1.25 \\
\hline 28 & 8.9 & 389.1080 & $\begin{array}{c}345.1166 ; 209.0438 ; 183.0653 \\
139.0066\end{array}$ & $\mathrm{C}_{16} \mathrm{H}_{22} \mathrm{O}_{11}$ & Deacetyl asperulosidic acid & 2.4 \\
\hline 29 & 9.1 & 373.1137 & $\begin{array}{c}193.0497 ; 167.0656 ; 149.0600 \\
123.0445 ; 105.0347\end{array}$ & $\mathrm{C}_{16} \mathrm{H}_{22} \mathrm{O}_{10}$ & Gardoside & 0.86 \\
\hline 30 & 9.7 & 595.1875 & $\begin{array}{c}549.1816 ; 225.0764 ; 207.0656 \\
123.0449\end{array}$ & $\mathrm{C}_{24} \mathrm{H}_{36} \mathrm{O}_{17}$ & Genipin gentiobioside & 0.79 \\
\hline 31 & 9.8 & 585.1591 & $549.1785 ; 225.0769$ & $\mathrm{C}_{36} \mathrm{H}_{26} \mathrm{O}_{8}$ & Genipin-1b-gentiobioside & -6.16 \\
\hline 32 & 10.1 & 353.0875 & $192.0590 ; 191.0556$ & $\mathrm{C}_{16} \mathrm{H}_{18} \mathrm{O}_{9}$ & cis-3-O-Caffeoylquinic acid & 0.86 \\
\hline 33 & 10.4 & 281.1386 & $\begin{array}{c}237.1456 ; 201.1270 ; 189.1279 \\
151.0740 ; 125.8689\end{array}$ & $\mathrm{C}_{15} \mathrm{H}_{22} \mathrm{O}_{5}$ & $\begin{array}{l}\text { 2-Carboxy-4-methyl-a-methylene-3-(3- } \\
\text { oxobutyl)-cyclohexaneacetic acid }\end{array}$ & 3.00 \\
\hline 34 & 10.7 & 433.1352 & $\begin{array}{c}225.0768 ; 207.0660 ; 123.0453 \\
101.0249\end{array}$ & $\mathrm{C}_{18} \mathrm{H}_{26} \mathrm{O}_{12}$ & Geniposide & -0.12 \\
\hline 35 & 10.8 & 337.0932 & $191.0543 ; 137.0253 ; 119.0498$ & $\mathrm{C}_{16} \mathrm{H}_{18} \mathrm{O}_{8}$ & 5-p-Coumaroylquinic acids & -0.91 \\
\hline 36 & 10.8 & 387.1302 & $\begin{array}{c}207.0659 ; 147.0443 ; 123.0448 ; \\
101.0247\end{array}$ & $\mathrm{C}_{17} \mathrm{H}_{24} \mathrm{O}_{10}$ & Vogeloside & -1.36 \\
\hline 37 & 11.3 & 403.1242 & $195.0660 ; 125.0241 ; 119.0350$ & $\mathrm{C}_{17} \mathrm{H}_{24} \mathrm{O}_{11}$ & Gardenoside & 0.95 \\
\hline 38 & 12.1 & 367.1038 & 191.0540; 173.0444 & $\mathrm{C}_{17} \mathrm{H}_{20} \mathrm{O}_{9}$ & 4-O-Caffeoylqunic methyl ester & -0.94 \\
\hline 39 & 12.1 & 515.1196 & $\begin{array}{c}353.0871 ; 335.0768 ; 191.0555 \\
179.0349 ; 135.0454\end{array}$ & $\mathrm{C}_{25} \mathrm{H}_{24} \mathrm{O}_{12}$ & 1,5-Dicaffeoylquinic acid & -0.19 \\
\hline
\end{tabular}


TABLE 1: Continued.

\begin{tabular}{|c|c|c|c|c|c|c|}
\hline $\begin{array}{l}\text { Peak } \\
\text { no. }\end{array}$ & $\begin{array}{c}\mathrm{RT} \\
(\mathrm{min}) \\
\end{array}$ & {$[\mathrm{M}-\mathrm{H}]^{-}$} & MS/MS fragmentation ions & Formula & Identification & ppm \\
\hline 40 & 12.7 & 375.1667 & $\begin{array}{c}285.1087 ; 193.0491 ; 173.0457 \\
135.0435 ; 119.0352\end{array}$ & $\mathrm{C}_{17} \mathrm{H}_{28} \mathrm{O}_{9}$ & Secologanic acid & -1.71 \\
\hline 41 & 12.6 & 367.1038 & 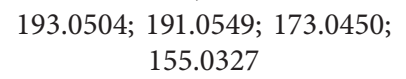 & $\mathrm{C}_{17} \mathrm{H}_{20} \mathrm{O}_{9}$ & 3-O-Caffeoylqunic methyl ester & -0.94 \\
\hline 42 & 12.9 & 497.1288 & $\begin{array}{c}353.0877 ; 233.0644 ; 191.0556 \\
173.0443 ; 161.0449\end{array}$ & $\mathrm{C}_{22} \mathrm{H}_{26} \mathrm{O}_{13}$ & $\begin{array}{l}\text { 3-O-(3-Hydroxy, 3-methyl)glutaroyl-4-O- } \\
\text { caffeoylquinic acid }\end{array}$ & 2.54 \\
\hline 43 & 13.8 & 403.1242 & $371.0974 ; 179.0674 ; 121.0292$ & $\mathrm{C}_{17} \mathrm{H}_{24} \mathrm{O}_{11}$ & Secoxyloganinc & 0.95 \\
\hline 44 & 14.1 & 519.1493 & $205.0487 ; 163.0393 ; 123.0445$ & $\mathrm{C}_{25} \mathrm{H}_{28} \mathrm{O}_{12}$ & $6^{\prime}$-O-trans-Coumaroyl geniposidic acid & 2.88 \\
\hline 45 & 14.2 & 497.1288 & $\begin{array}{c}353.0853 ; 233.0623 ; 191.0554 \\
179.0330 ; 135.0445\end{array}$ & $\mathrm{C}_{22} \mathrm{H}_{26} \mathrm{O}_{13}$ & $\begin{array}{l}\text { 3-O-Caffeoyl-4-O-(3-hydroxy, 3-methyl) } \\
\text { glutaroylquinic acid }\end{array}$ & 2.54 \\
\hline 46 & 14.3 & 389.1080 & 181.0495 & $\mathrm{C}_{16} \mathrm{H}_{22} \mathrm{O}_{11}$ & Deacetyl asperulosidic acid & 2.4 \\
\hline 47 & 14.4 & 529.1355 & $367.1019 ; 193.0492 ; 173.0450$ & $\mathrm{C}_{26} \mathrm{H}_{26} \mathrm{O}_{12}$ & 3,5-di-O-Caffeoylquinic methyl ester & -0.66 \\
\hline 48 & 15.2 & 279.123 & $\begin{array}{l}205.1219 ; 187.1107 ; 139.0757 \\
119.0848\end{array}$ & $\mathrm{C}_{15} \mathrm{H}_{20} \mathrm{O}_{5}$ & Artemisitene & 2.85 \\
\hline 49 & 15.1 & 463.0868 & 301.0330 & $\mathrm{C}_{21} \mathrm{H}_{20} \mathrm{O}_{12}$ & Quercetin-3-O-glucoside & 0.09 \\
\hline 50 & 15.1 & 609.144 & 301.0341 & $\mathrm{C}_{27} \mathrm{H}_{30} \mathrm{O}_{16}$ & Rutin & 3.46 \\
\hline 51 & 15.2 & 281.1389 & $\begin{aligned} 251.1282 ; & 207.1365 ; 179.1425 \\
& 137.0991\end{aligned}$ & $\mathrm{C}_{15} \mathrm{H}_{22} \mathrm{O}_{5}$ & Artemisinin & 1.95 \\
\hline 52 & 15.6 & 447.0926 & $285.0391 ; 447.0919$ & $\mathrm{C}_{21} \mathrm{H}_{20} \mathrm{O}_{11}$ & Luteolin-7-O-D-glucopyranoside & 1.53 \\
\hline 53 & 16.3 & 593.1500 & 285.0405 & $\mathrm{C}_{27} \mathrm{H}_{30} \mathrm{O}_{15}$ & Nicotiflorin & 2.01 \\
\hline 54 & 16.2 & 515.1196 & $\begin{aligned} 135.0440 ; & 179.0330 ; 191.0557 ; \\
& 353.0864\end{aligned}$ & $\mathrm{C}_{25} \mathrm{H}_{24} \mathrm{O}_{12}$ & 1,3-Dicaffeoylquinicacid & -0.19 \\
\hline 55 & 16.3 & 519.1493 & $\begin{array}{c}123.0453 ; 145.0256 ; 163.0387 \\
307.0815 ; 193.05\end{array}$ & $\mathrm{C}_{25} \mathrm{H}_{28} \mathrm{O}_{12}$ & 6-O-trans-Coumaroyl geniposidic acid & 2.88 \\
\hline 56 & 16.4 & 429.1394 & $\begin{array}{c}101.0251 ; 249.0766 ; 205.0816 \\
191.0766 ; 173.0558\end{array}$ & $\mathrm{C}_{19} \mathrm{H}_{26} \mathrm{O}_{11}$ & 10-Acetyl geniposide & 1.94 \\
\hline 57 & 16.9 & 515.1197 & $\begin{array}{c}353.0875 ; 191.0557 ; 179.0347 \\
135.0446\end{array}$ & $\mathrm{C}_{25} \mathrm{H}_{24} \mathrm{O}_{12}$ & 3,4-Dicaffeoylquinic acid & -0.39 \\
\hline 58 & 17.2 & 515.1196 & $\begin{array}{c}353.0875 ; 191.0554 ; 179.0343 \\
161.0237 ; 135.0449\end{array}$ & $\mathrm{C}_{25} \mathrm{H}_{24} \mathrm{O}_{12}$ & 3,5-Dicaffeoylquinic acid & -0.19 \\
\hline 59 & 18.7 & 515.1196 & $\begin{array}{c}353.0879 ; 191.0555 ; 179.0345 \\
135.0448\end{array}$ & $\mathrm{C}_{25} \mathrm{H}_{24} \mathrm{O}_{12}$ & 4,5-Dicaffeoylquinic acid & -0.19 \\
\hline 60 & 18.9 & 723.2134 & $\begin{array}{c}677.4951 ; 353.0892 ; 335.0760 \\
191.0567\end{array}$ & $\mathrm{C}_{33} \mathrm{H}_{40} \mathrm{O}_{18}$ & Jasmigeniposide A & 1.09 \\
\hline 61 & 19.1 & 515.1196 & $\begin{array}{c}353.0873 ; 191.0565 ; 179.0344 \\
135.0446\end{array}$ & $\mathrm{C}_{25} \mathrm{H}_{24} \mathrm{O}_{12}$ & 1,4-Dicaffeoylquinic acid & -0.19 \\
\hline 62 & 19.1 & 757.2548 & $\begin{array}{c}595.2013 ; 525.1608 ; 493.1693 \\
179.0574 ; 161.0443\end{array}$ & $\mathrm{C}_{34} \mathrm{H}_{46} \mathrm{O}_{19}$ & Japonicaside A & 1.65 \\
\hline 63 & 19.1 & 803.2601 & $\begin{array}{c}595.2014 ; 493.1700 ; 179.0551 \\
161.0439\end{array}$ & $\mathrm{C}_{35} \mathrm{H}_{48} \mathrm{O}_{21}$ & Centauroside & 1.78 \\
\hline 64 & 19.2 & 695.2179 & $\begin{array}{c}469.1398 ; 225.0744 ; 163.0396 \\
145.0306\end{array}$ & $\mathrm{C}_{32} \mathrm{H}_{40} \mathrm{O}_{17}$ & $6^{\prime}$-O-trans-Coumaroyl genipin gentiobioside & 1.97 \\
\hline 65 & 19.6 & 659.1607 & $\begin{array}{c}497.1296 ; 353.0869 ; 335.0855 \\
191.0543 ; 161.0439\end{array}$ & $\mathrm{C}_{31} \mathrm{H}_{32} \mathrm{O}_{17}$ & $\begin{array}{l}\text { 3,5-di-O-Caffeoyl-4-O-(3-hydroxy-3-methyl)- } \\
\text { glutaroylquinic acid }\end{array}$ & -1.6 \\
\hline 66 & 19.7 & 755.2380 & $529.1525 ; 225.0767 ; 123.0449$ & $\mathrm{C}_{34} \mathrm{H}_{44} \mathrm{O}_{19}$ & $6^{\prime \prime}$-O-trans-Sinapoyl genipin gentiobioside & 3.18 \\
\hline 67 & 19.8 & 725.2277 & $\begin{array}{c}499.1413 ; 225.0751 ; 123.0450 \\
101.0235\end{array}$ & $\mathrm{C}_{33} \mathrm{H}_{42} \mathrm{O}_{18}$ & $6^{\prime \prime}$-O-trans-Cinnamoyl genipin gentiobioside & 2.94 \\
\hline
\end{tabular}

$\left.\mathrm{C}_{6} \mathrm{H}_{12} \mathrm{O}_{6}-\mathrm{CO}_{2}-2 \mathrm{H}_{2} \mathrm{O}\right]^{-}$. This behavior is completely identical with the property of the 2-carboxy-4-methyl-amethylene-3-(3-oxobutyl)-cyclohexaneacetic acid. Peak 36 were located at $10.8 \mathrm{~min}$ in the extracted ion chromatogram and they produced a precursor ion at $\mathrm{m} / \mathrm{z} 387.1302$. It was tentatively assigned as vogeloside because it had fragment ions 207.0659 [M-H- $\left.\mathrm{C}_{6} \mathrm{H}_{12} \mathrm{O}_{6}\right]^{-}, 147.0443$ [M-H- $\mathrm{C}_{6} \mathrm{H}_{12} \mathrm{O}_{6^{-}}$ $\left.\mathrm{CH}_{3} \mathrm{COOH}\right]^{-}$, and $\left[\mathrm{M}-\mathrm{H}-\mathrm{C}_{6} \mathrm{H}_{12} \mathrm{O}_{6}-\mathrm{CH}_{3} \mathrm{COOH}-\mathrm{HCOOH}\right]$ [39]. The total ion chromatography of 67 components from $\mathrm{RDN}$ injection is shown in Figure 3. In order to further confirm the main components from RDN injection, the reference standards were injected to UHPLC-Q-TOF/MS. As shown in Table 2, the precursor ions and MS/MS fragmentation ions of neochlorogenic acid, chlorogenic acid, cryptochlorogenic acid, isochlorogenic acid $\mathrm{B}$, isochlorogenic acid $\mathrm{A}$, and isochlorogenic acid $\mathrm{C}$ were the same as those in RDN injection (peaks 5, 20, 25, 57, 58, and 59), which gave the definite evidence to support the results of identification. The total ion chromatography of reference standards is shown in Figure 4. 




FIgURE 3: UHPLC/Q-TOF-MS chromatography of RDN injection in a negative mode.

TABLE 2: UPLC/Q-TOF-MS data of main active compounds from RDN injection.

\begin{tabular}{lcccccc}
\hline Peak no. & RT $(\min )$ & {$[\mathrm{M}-\mathrm{H}]-$} & MS/MS fragmentation ion & Formula & Identification & ppm \\
\hline 1 & 2.680 & 353.0884 & $191.0550 ; 179.0349 ; 135.0454$ & $\mathrm{C}_{16} \mathrm{H}_{18} \mathrm{O}_{9}$ & Neochlorogenic acid & -1.6 \\
2 & 5.848 & 353.0872 & $191.0571 ; 179.0301 ; 135.0311$ & $\mathrm{C}_{16} \mathrm{H}_{18} \mathrm{O}_{9}$ & Chlorogenic acid & 1.7 \\
3 & 7.522 & 353.0883 & $191.0560 ; 179.0357 ; 135.0444$ & $\mathrm{C}_{16} \mathrm{H}_{8} \mathrm{O}_{9}$ & Cryptochlorogenic acid & -1.4 \\
4 & 15.067 & 515.1185 & $353.0887 ; 191.0561 ; 179.0367 ; 135.0454$ & $\mathrm{C}_{25} \mathrm{H}_{24} \mathrm{O}_{12}$ & Isochlorogenic acid B & 1.94 \\
5 & 15.249 & 515.1209 & $353.0872 ; 191.0570 ; 179.0345 ; 135.0434$ & $\mathrm{C}_{25} \mathrm{H}_{24} \mathrm{O}_{12}$ & Isochlorogenic acid A & 2.71 \\
6 & 16.559 & 515.1203 & $353.0874 ; 191.0575 ; 179.0330 ; 135.0423$ & $\mathrm{C}_{25} \mathrm{H}_{24} \mathrm{O}_{12}$ & Isochlorogenic acid C & -1.55 \\
\hline
\end{tabular}

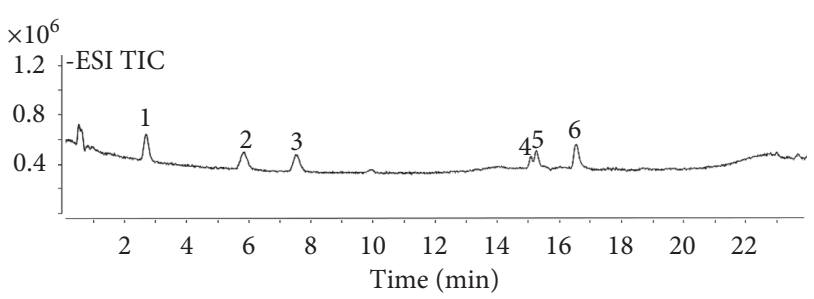

FIgURE 4: UHPLC/Q-TOF-MS chromatography of main active components from RDN injection. 1: neochlorogenic acid; 2: chlorogenic acid; 3: cryptochlorogenic acid; 4: isochlorogenic acid B; 5: isochlorogenic acid A; and 6: isochlorogenic acid C.

3.3. Screening the Potential Active Fractions of NIs. An in vitro activity assay was performed to screen the potential NAIs. Only fractions with dose-effect relationships were thought of as potential active components, which were identified and validated as the NAIs. Some components had NA inhibition just for only one concentration but did not exhibit dose-effect relationship and therefore could not be selected as active components. On the other hand, some fractions showed the inhibition with dose-effect relationships, but the inhibition rates of each concentration were just below the limit activity at $6 \%$. Hence, they were not selected as active components. The reason why the rate was chosen at $6 \%$ was that the systematic error was $6 \%$ obtained by the calibration curve for screening the NAIs. In total, the inhibitory ratios of 28 fractions which were truly active fractions among the 75 fractions (fractions $3,4,5,6,8,15,18,19,20,21,22,23,25,29,30,31,32$, $38,39,40,46,47,66,67,68,69,70$, and 71) increased with an increasing concentration, which exhibited obvious relativity between the dose and the effect as shown in Figure 5. These fractions have been indicated as the potential NAIs.
3.4. Verifying the Potential Active Fractions via UHPLC and UHPLC-Q-TOF/MS. A total of 75 fractions were prepared. After screening the inhibitors, each fraction was injected to the UHPLC again. Comparing each fraction with RDN injection, the components from each fraction can be primarily identified depending on their retention time and corresponding peak number. The results can be shown in Figure 6 . The fractions $(3,4,5,6,8,15,18,19,20,21,23,25$, $27,29,30,31,32,38,39,40,46,47,66,67,68,69,70$, and 71) contained peaks which have the same retention time with RDN injection. Finally, the corresponding relationship between fraction number and peak number of the potential active components with NA inhibition is shown in Table 3.

3.5. Confirmation of the Main Components with NA Inhibition. In order to confirm the bioactivity of the components, the main active components with NAIs rates reaching 50\% including 6 components, neochlorogenic acid, chlorogenic acid, cryptochlorogenic acid, isochlorogenic acid $\mathrm{B}$, isochlorogenic acid $\mathrm{A}$, and isochlorogenic acid $\mathrm{C}$, were selected to be further validated. $\mathrm{IC}_{50}$ value was determined to be the effective concentration at which the activity of NA was inhibited by $50 \%$. As a result, the $\mathrm{IC}_{50}$ value of neochlorogenic acid, chlorogenic acid, cryptochlorogenic acid, isochlorogenic acid $\mathrm{B}$, isochlorogenic acid $\mathrm{A}$, and isochlorogenic acid $\mathrm{C}$ were calculated as $23.79 \mu \mathrm{g}$ $\mathrm{mL}^{-1}, 17.40 \mu \mathrm{g} \mathrm{mL}^{-1}, 231.7 \mu \mathrm{g} \mathrm{mL}^{-1}, 40.33 \mu \mathrm{g} \mathrm{mL}^{-1}$, less than $10 \mu \mathrm{g} \mathrm{mL}{ }^{-1}$, and $14.52 \mu \mathrm{g} \mathrm{mL}^{-1}$, respectively. Studying it further, the total inhibition ratio of these 6 components has reached almost $80 \%$ of RDN injection. As shown in Figure 7, comparing the inhibition ratio of $\mathrm{RDN}$ injection and 6 components whose concentrations were the same with RDN injection, it was found that the inhibition ratios of screening 6 active components and RDN injection were almost the 

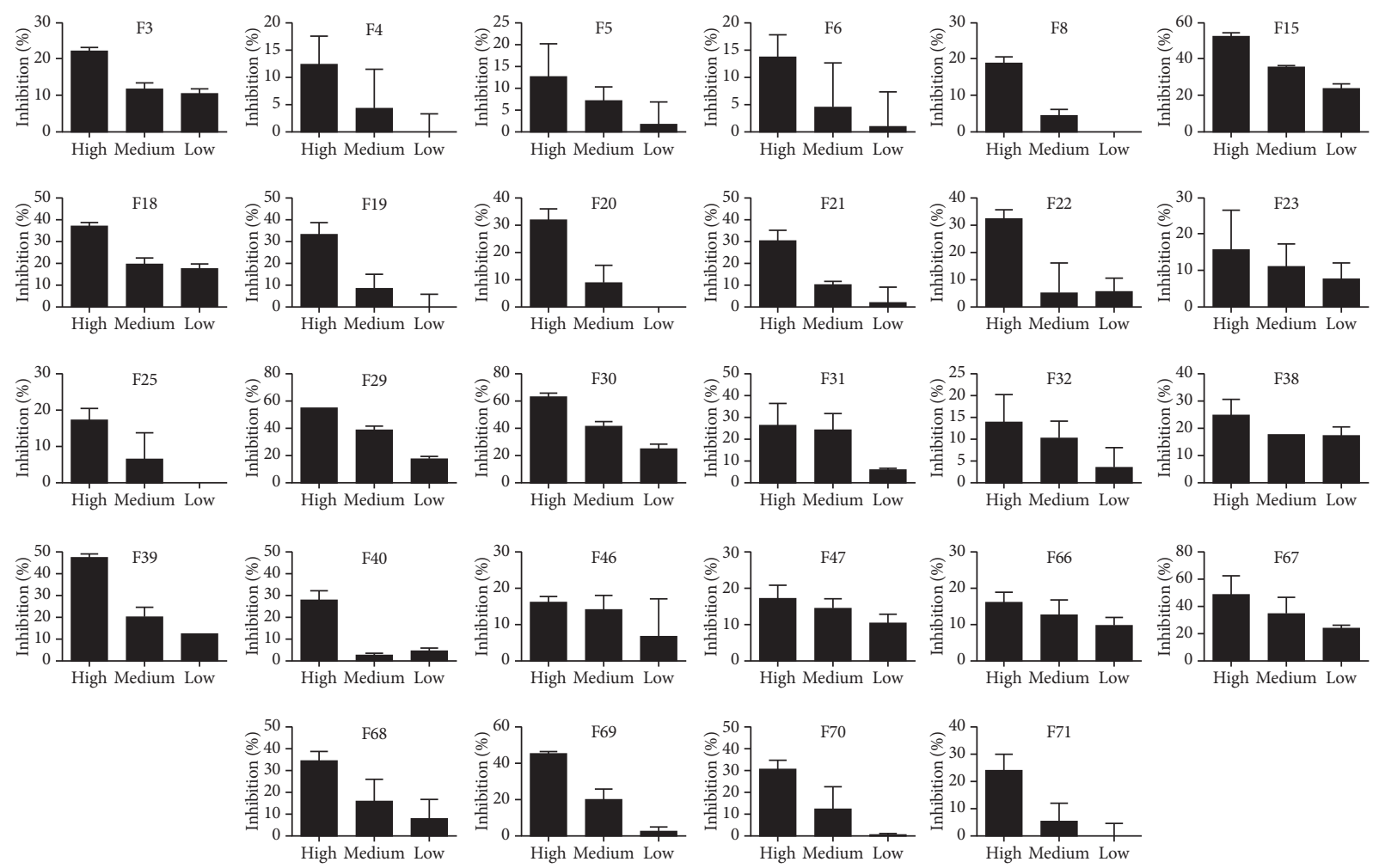

FIgURE 5: The 28 potential active fractions showed the NA inhibition with dose-effect relationships.

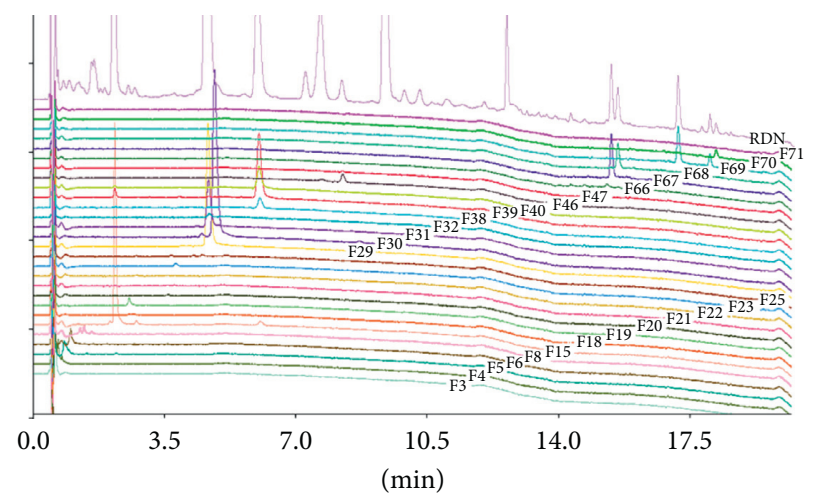

FIGURE 6: Comparing the chromatography of 28 potential active fractions with RDN injection.

same, indicating that the screening 6 active components could reflect the total inhibition ratio of RDN injection against NA. As shown in Figure 7, the inhibition ratios of $\mathrm{RDN}$ injection with different dilution times and 6 components seemed to have similar property. Moreover, these components contained the original nucleus of quininic acid and caffeic acid, from which it could be concluded that chlorogenic acids were main active components against NA.

3.6. Comparison of the Developed Method and Others. Nowadays, many researchers focused on the screening active components from TCM. It is therefore essential to establish the direct, rapid, and accurate methods for screening these active components. A great number of screening methods were based on only one concentration of components for evaluating the biological activity. However, these methods have not been proven to evaluate the dose-effect activity of the selected components. In our study, the developed method combined the isolation, screening, and identification of bioactive compounds using the HPLC-FC for the preparation and isolation while using UHPLC and UHPLC/ Q-TOF-MS for identifying. More importantly, three different concentrations (low, medium, and high concentration) were prepared by one-step HPLC-FC method for screening the active components, making the selected active compounds more credible via dose-effect relationship. What is more, the developed method can also identify other components from RDN injection. Therefore, the developed method was not only accurate to identify 67 compounds 
TABLE 3: The corresponding relationship between fraction number and peak number of the potential active components with NA inhibition.

\begin{tabular}{lcc}
\hline Fraction number & Peak number & Identification \\
\hline $3,4,5$ & 1 & Quinic acid \\
6 & 2 & Geniposidic acid \\
8 & 3 & Shanzhiside \\
15 & 5 & Neochlorogenic acid \\
18,19 & 8 & Jasminoside \\
20 & 13,14 & Scandoside methyl ester, 3-p-coumaroylquinic acids \\
21 & 14,15 & 3-p-Coumaroylquinic acids, vanillin \\
22 & 15 & Vanillin \\
23,25 & 16 & Cir-1-O-caffeoylquinic acid \\
$29,30,31,32$ & Chlorogenic acid \\
$38,39,40$ & 20 & Cryptochlorogenic acid \\
46,47 & 25 & Genipin gentiobioside \\
66,67 & 30 & Isochlorogenic acid B \\
68 & 57 & Isochlorogenic acid A \\
69 & 58 & Isochlorogenic acid C \\
70,71 & 59 & 1,4 -Dicaffeoylquinic acid \\
\hline
\end{tabular}

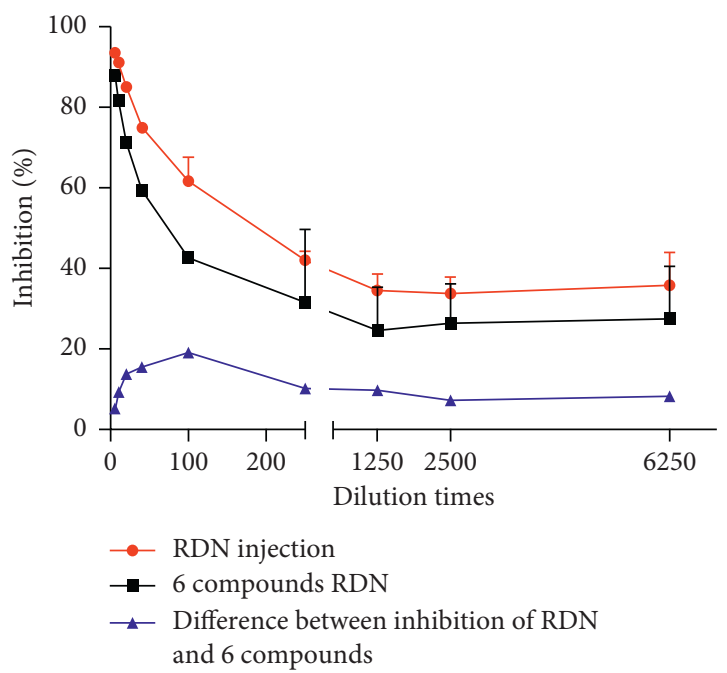

FIgURE 7: The inhibition ratio of RDN injection and 6 active components combination with different dilution times and the difference between inhibition of $\mathrm{RDN}$ and 6 components combination.

from RDN injection, but also credible for directly screening NAIs compounds from RDN injection, which provides a promising paradigm for elucidating the bioactive constituents in TCM.

\section{Conclusions}

An accurate method was successfully developed to screen NAIs from RDN injection by using HPLC-FC for preparation and isolation and UHPLC/Q-TOF-MS for identification. Three different concentrations (low, medium, and high concentration) of each fraction were prepared by only one injection, which can be validated for screening active components with the dose-effect relationship. In total, 67 components were identified from RDN injection and 15 active components showed an inhibitory effect against NA with a dose-effect relationship. Moreover, it was worth noting that neochlorogenic acid, chlorogenic acid, cryptochlorogenic acid, isochlorogenic acid $\mathrm{B}$, isochlorogenic acid $\mathrm{A}$, isochlorogenic acid $\mathrm{C}$, and quinic acid were the main active components that accounted for almost $80 \%$ of inhibitory activity of RDN injection. Accordingly, an activity-integrated HPLC-FC and UHPLC/ Q-TOF-MS strategy was as not only accurate to identify the components from TCM, but also credible and economic for directly screening active components, which provided a promising paradigm for elucidating the bioactive components in TCM.

\section{Data Availability}

The data are included within the manuscript.

\section{Conflicts of Interest}

The authors declare that they have no conflicts of interest regarding the publication of this paper.

\section{Authors' Contributions}

Conceptualization of the study was done by Yan-Xu Chang and Jin Li. Data curation was obtained by Lijun An and XieAn Yu. Formal analysis was performed by Lijun An, Xie-An $\mathrm{Yu}$, and Wei Liu. Funding acquisition was supported by Yan-Xu Chang. Lijun An and Xie-an Yu wrote the original draft. Jin Li and Yan-xu Chang reviewed and edited the draft. Lijun An and Xie-An Yu have contributed equally to this work.

\section{Acknowledgments}

This research was supported by the National Natural Science Foundation of China (no. 81374050), Tianjin Research Program of Application Foundation and Advanced Technology (no. 18JCYBJC95000), and Special Program of Talents Development for Excellent Youth Scholars in Tianjin. 


\section{References}

[1] F. Krammer and P. Palese, "Advances in the development of influenza virus vaccines," Nature Reviews Drug Discovery, vol. 14, no. 3, pp. 167-182, 2015.

[2] A. B. Ryder, L. Buonocore, L. Vogel, R. Nachbagauer, F. Krammer, and J. K. Rose, "A viable recombinant rhabdovirus lacking its glycoprotein gene and expressing influenza virus hemagglutinin and neuraminidase is a potent influenza vaccine," Journal of Virology, vol. 89, no. 5, pp. 2820-2830, 2015.

[3] Y. H. Baek, M.-S. Song, E.-Y. Lee et al., "Profiling and characterization of influenza virus N1 strains potentially resistant to multiple neuraminidase inhibitors," Journal of $\mathrm{Vi}$ rology, vol. 89, no. 1, pp. 287-299, 2015.

[4] F. M. Cardoso, L. I. Ibañez, S. Van den Hoecke et al., "Singledomain antibodies targeting neuraminidase protect against an H5N1 influenza virus challenge," Journal of Virology, vol. 88, p. 15, 2015.

[5] A. Meijer, H. Rebelo-de-Andrade, V. Correia et al., "Global update on the susceptibility of human influenza viruses to neuraminidase inhibitors, 2012-2013," Antiviral Research, vol. 110, pp. 31-41, 2014.

[6] J. R. Westin, F. Chu, M. Zhang et al., "Safety and activity of PD1 blockade by pidilizumab in combination with rituximab in patients with relapsed follicular lymphoma: a single group, open-label, phase 2 trial," The Lancet Oncology, vol. 15, no. 1, pp. 69-77, 2014.

[7] N. Sriwilaijaroen, K. Suzuki, E. Takashita, H. Hiramatsu, O. Kanie, and Y. Suzuki, "6SLN-lipo PGA specifically catches (coats) human influenza virus and synergizes neuraminidasetargeting drugs for human influenza therapeutic potential," Journal of Antimicrobial Chemotherapy, vol. 70, no. 10, pp. 2797-2809, 2015.

[8] H. F. Wu, S. L. Morris-Natschke, X. D. Xu et al., "Recent advances in natural anti-HIV triterpenoids and analogs," Medicinal Research Reviews, pp. 1-47, 2020.

[9] J.-M. Pawlotsky, "Hepatitis C virus resistance to direct-acting antiviral drugs in interferon-free regimens," Gastroenterology, vol. 151, no. 1, pp. 70-86, 2016.

[10] M. Hussain, H. Galvin, T. Y. Haw, A. Nutsford, and M. Husain, "Drug resistance in influenza A virus: the epidemiology and management," Infection and Drug Resistance, vol. 10, p. 121, 2017.

[11] L.-P. Tang, Z.-F. Mao, X.-X. Li et al., "ReDuNing, a patented Chinese medicine, reduces the susceptibility to H1N1 influenza of mice loaded with restraint stress," European Journal of Integrative Medicine, vol. 6, no. 6, pp. 637-645, 2014.

[12] Y. X. Chang, J. Liu, Y. Bai, J. Li, E. W. Liu, and J. He, “The activity-integrated method for quality assessment of reduning injection by on-line DPPH-CE-DAD," PLOS ONE, vol. 9, Article ID e106254, 2014.

[13] H. Yang, W. Zhang, C. Huang et al., "A novel systems pharmacology model for herbal medicine injection: a case using reduning injection," BMC Complementary and Alternative Medicine, vol. 14, p. 430, 2014.

[14] J. Liu, K. Sun, C. Zheng, X. Chen, W. Zhang, and Z. Wang, "Pathway as a pharmacological target for herbal medicines: an investigation from reduning injection," PLoS One, vol. 10, Article ID e0123109, 2015.

[15] F. Luo, J. Gu, X. Zhang, L. Chen, L. Cao, and N. Li, "Multiscale modeling of drug-induced effects of ReDuNing injection on human disease: from drug molecules to clinical symptoms of disease," Scientific Reports, vol. 5, Article ID 10064, 2015.
[16] A.-h. Ge, Y. Bai, J. Li et al., "An activity-integrated strategy involving ultra-high-performance liquid chromatography/ quadrupole-time-of-flight mass spectrometry and fraction collector for rapid screening and characterization of the $\alpha$-glucosidase inhibitors in Coptis chinensis Franch. (Huanglian)," Journal of Pharmaceutical and Biomedical Analysis, vol. 100, pp. 79-87, 2014.

[17] J. Lu, H.-P. Song, P. Li, P. Zhou, X. Dong, and J. Chen, "Screening of direct thrombin inhibitors from Radix Salviae Miltiorrhizae by a peak fractionation approach," Journal of Pharmaceutical and Biomedical Analysis, vol. 109, pp. 85-90, 2015.

[18] H. Zhao and Z. Chen, "Screening of neuraminidase inhibitors from traditional Chinese medicine by transverse diffusion mediated capillary microanalysis," Biomicrofluidics, vol. 8, Article ID 052003, 2014.

[19] Z. Fu, Y. Ling, Z. Li, M. Chen, Z. Sun, and C. Huang, "HPLCQ-TOF-MS/MS for analysis of major chemical constituents of Yinchen-Zhizi herb pair extract," Biomedical Chromatography, vol. 28, no. 4, pp. 475-485, 2014.

[20] J.-Y. Zhang, Q. Zhang, N. Li, Z.-J. Wang, J.-Q. Lu, and Y.-J. Qiao, "Diagnostic fragment-ion-based and extension strategy coupled to DFIs intensity analysis for identification of chlorogenic acids isomers in Flos Lonicerae Japonicae by HPLC-ESI-MSn," Talanta, vol. 104, pp. 1-9, 2013.

[21] S. Zhang, Y.-J. Li, C.-X. Zhang et al., "Research on the change of chemical composition in productive process of Re Du Ning injection by HPLC/Q-TOF MS," Biomedical Chromatography, vol. 30, no. 2, pp. 131-141, 2016.

[22] M. N. Clifford, W. Wu, J. Kirkpatrick, R. Jaiswal, and N. Kuhnert, "Profiling and characterisation by liquid chromatography/multi-stage mass spectrometry of the chlorogenic acids in Gardeniae Fructus," Rapid Communications in Mass Spectrometry, vol. 24, no. 21, pp. 3109-3120, 2010.

[23] L. Yang, H. Jiang, X. Xing et al., "A biosensor-based quantitative analysis system of major active ingredients in Lonicera japonica thunb. Using UPLC-QDa and chemometric analysis," Molecules, vol. 24, no. 9, p. 1787, 2019.

[24] J. Liu, D. Yuan, J. Ding, X. Dong, G. Dou, and Z. Wu, "Study on the metabolites of 1,5-dicaffeoylquinic acid in human urine by LC-ESI/MS/MS assay," Chin J Clin Pharmacol, vol. 26, pp. 217-220, 2010.

[25] A. Vinayagam and P. Sudha, "Separation and identification of phenolic acid and flavonoids from Nerium indicum flowers," Indian Journal of Pharmaceutical Sciences, vol. 77, no. 1, p. 91, 2015.

[26] R. Jaiswal, H. Müller, A. Müller, M. G. E. Karar, and N. Kuhnert, "Identification and characterization of chlorogenic acids, chlorogenic acid glycosides and flavonoids from Lonicera henryi L. (Caprifoliaceae) leaves by LC-MSn," Phytochemistry, vol. 108, pp. 252-263, 2014.

[27] Z. Fu, R. Xue, Z. Li et al., "Fragmentation patterns study of iridoid glycosides in Fructus Gardeniae by HPLC-Q/TOFMS/MS," Biomedical Chromatography, vol. 28, no. 12, pp. 1795-1807, 2014.

[28] M. J. Son, C. Jin, Y. S. Lee, J. Y. Lee, and H. J. Kim, "Characterization of caffeoylglucoside derivatives and hypouricemic activity of the ethyl acetate fraction from aster glehni," Bulletin of the Korean Chemical Society, vol. 36, pp. 503-512, 2015.

[29] H. J. Kim, E. J. Kim, S. H. Seo, C.-G. Shin, C. Jin, and Y. S. Lee, "Vanillic acid glycoside and quinic acid derivatives from Gardeniae Fructus," Journal of Natural Products, vol. 69, no. 4, pp. 600-603, 2006. 
[30] C. Bastos, L. Barros, M. Dueñas et al., "Chemical characterisation and bioactive properties of Prunus avium L.: the widely studied fruits and the unexplored stems," Food Chemistry, vol. 173, pp. 1045-1053, 2015.

[31] L. Zhang, Y.-L. Ma, Y. Liu, and Y.-G. Zu, "Development and validation of high liquid performance chromatography-tandem mass spectrometry method for simultaneous determination of geniposidic acid and aucubin in rat plasma for pharmacokinetic study after oral administration of Du-zhong tea extract," Journal of Chromatography B, vol. 963, pp. 62-69, 2014.

[32] H.-B. Li, Y. Yu, Y.-D. Mei et al., "A new hetero dimeric terpenoid derivative, japonicaside $\mathrm{C}$, from the flower buds of Lonicera japonica," Natural Product Research, vol. 31, no. 2, pp. 143-148, 2017.

[33] S. Sharma, B. D. Gupta, R. Kant, and V. K. Gupta, "Synthesis, characterization, and crystal structure of negundoside ( $2^{\prime}$-phydroxybenzoyl mussaenosidic acid)," Journal of Crystallography, vol. 2014, pp. 1-6, 2014.

[34] K. Yonekura-Sakakibara, R. Nakabayashi, S. Sugawara, T. Tohge, T. Ito, and M. Koyanagi, "A flavonoid 3-O-glucoside: $2^{\prime \prime}$-O-glucosyltransferase responsible for terminal modification of pollen-specific flavonols in Arabidopsis thaliana," The Plant Journal, vol. 79, pp. 769-782, 2014.

[35] Y.-P. Shi, Y.-G. Zhang, H.-N. Li et al., "Discovery and identification of antithrombotic chemical markers in Gardenia Fructus by herbal metabolomics and zebrafish model," Journal of Ethnopharmacology, vol. 253, Article ID 112679, 2020.

[36] H. Liu, Y.-F. Chen, F. Li, and H.-Y. Zhang, "Fructus Gardenia (Gardenia jasminoides J. Ellis) phytochemistry, pharmacology of cardiovascular, and safety with the perspective of new drugs development," Journal of Asian Natural Products Research, vol. 15, no. 1, pp. 94-110, 2013.

[37] V. Salim, F. Yu, J. Altarejos, and V. De Luca, "Virus-induced gene silencing identifiesCatharanthus roseus7-deoxyloganic acid-7-hydroxylase, a step in iridoid and monoterpene indole alkaloid biosynthesis," The Plant Journal, vol. 76, no. 5, pp. 754-765, 2013.

[38] L.-1. Wu, Y.-S. Wu, W.-Y. Chen et al., "Determination of artemisitene in rat plasma by ultra-performance liquid chromatography/tandem mass spectrometry and its application in pharmacokinetics," Rapid Communications in Mass Spectrometry, vol. 31, no. 13, pp. 1121-1128, 2017.

[39] L. N. Wang, Q. Jiang, J. H. Hu, Y. Q. Zhang, and J. Li, "Research progress on chemical constituents of Lonicerae japonicae flos," BioMed Research International, vol. 2016, Article ID 8968940, , 2016. 